\title{
PROPUESTA DE ARQUITECTURA EMPRESARIAL PARA EL GRUPO DE PERFORACIÓN DE ECOPETROL ICP USANDO EL MARCO DE REFERENCIA TOGAF
}

\author{
Javier Eduardo Gelvis Vega', Sebastián Roa Prada², Claudia Isabel Cáceres Becerra ${ }^{3}$, \\ Hernán Darío Mantilla Hernandez ${ }^{4}$
}

${ }^{1}$ Ingeniero de sistemas. Magister en gestión, aplicación y desarrollo de software. Universidad Autónoma de Bucaramanga. jgelvis@unab.edu.co.

2Doctor en Ingeniería Mecánica. Docente. Universidad Autónoma de Bucaramanga. sroa@unab.edu.co

3Master en gestión, aplicación y desarrollo de software, Docente. Universidad Autónoma de Bucaramanga. ccaceres4@unab.edu.co

${ }^{4}$ Master en Geotecnia. Ingeniero de Perforación. Ecopetrol. hernan.mantilla@ecopetrol.com.co.

\section{RESUMEN}

La arquitectura empresarial permite armonizar los componentes tecnológicos de una organización definiendo un norte acorde con los objetivos y estrategias corporativas. En el grupo de geomecánica y perforación del ICP se quiere contar con elementos que sean de apoyo para generar desarrollos de herramientas software que permitan monitorear la perforación de pozos petroleros en tiempo real. Este trabajo presenta un marco conceptual de arquitectura empresarial enfocado en la optimización del flujo de información para apoyar la toma de decisiones estratégicas, generando una hoja de ruta de desarrollo de herramientas software que faciliten la gestión de información para el grupo de geomecánica del ICP. En el diseño de la arquitectura empresarial se trabajó en las seis primeras fases de la metodología ADM que proporciona TOGAF.

Palabras clave: Arquitectura Empresarial; TOGAF; Gestión de información, Hoja de ruta

Recibido: 12 de diciembre de 2020. Aceptado: 06 de marzo de 2021

Received: December 12, 2020. Accepted: March 06, 2021

DOI: https://doi.org/10.33571/rpolitec.v17n33a2

\section{ENTERPRISE ARCHITECTURE PROPOSAL FOR ECOPETROL-ICP DRILLING GROUP USING TOGAF FRAMEWORK}

\begin{abstract}
Enterprise architecture allows to harmonize a company's technological components by defining a north star according to corporate goals and strategies. Ecopetrol-ICP's geomechanics group needs to have elements to support software development for real time drilling monitoring. This work presents a conceptual framework for enterprise architecture focused on the optimization of information flow to support strategic decision-making by creating a software development roadmap to ease IT management for ICP's geomechanics research group. In the Enterprise Architecture design with TOGAF the first six phases of the ADM cycle were implemented.
\end{abstract}

Keywords: Enterprise architecture, TOGAF, IT management, roadmap

Cómo citar este artículo: J.E. Gelvis-Vega, S. Roa-Prada, C.I. Cáceres-Becerra, H. Mantilla. "Propuesta de arquitectura empresarial para el grupo de perforación de Ecopetrol ICP usando el marco de referencia TOGAF", Revista Politécnica, vol.17, no.33 pp.22-33, 2021. DOI: https://doi.org/10.33571/rpolitec.v17n33a2 


\section{INTRODUCCIÓN}

Un alto porcentaje del petróleo producido a nivel mundial proviene de campos de petróleo en un estado maduro y constituyen una oportunidad para suplir la necesidad energética mundial creciente [1].

La volatilidad de los precios del petróleo introduce una gran incertidumbre en el desarrollo de las actividades productivas de las empresas petroleras. Dado que los precios del petróleo son determinados por factores de oferta y demanda en el mercado mundial, es necesario que las empresas petroleras aumenten la eficiencia de sus procesos continuamente para poderse ajustar, y mantenerse activos en el negocio, incluso en las condiciones económicas más desfavorables. Los procesos productivos de la industria petrolera, como por ejemplo la perforación, dependen de una gran cantidad de variables [2]. En algunos casos es posible que no se tenga a disposición toda la información de las variables de proceso, en otros casos se puede llegar a contar con parte de la información. Entonces, se vuelve muy importante poder aprovechar esa información, procesarla, y extraer conocimiento que pueda ser usado como apoyo en la toma de decisiones que mejoren la eficiencia de procesos y la competitividad de la empresa.

Para el grupo de perforación del ICP es muy importante mantener las herramientas de análisis y las bases de datos de información de perforación actualizadas y poder realizar las tareas de análisis de datos con información de alta calidad, cumpliendo con los lineamientos definidos por la Vicepresidencia Digital de Ecopetrol [3][4].

La Arquitectura Empresarial (AE) es una metodología que tiene en cuenta la visión integral de la organización y permite alinear los diferentes dominios (procesos de negocio, datos, aplicaciones y tecnología) con los objetivos del negocio.

Un ejercicio de $\mathrm{AE}$ ayuda a establecer un plan estratégico en cualquier organización, guiado por los componentes de negocio y los sistemas de información. Permite conocer el estado ideal al que quiere llegar la organización, usando tecnologías para soportar los procesos necesarios para alcanzarlo.

El presente trabajo tiene como finalidad suministrar un marco conceptual de arquitectura empresarial enfocado en la optimización del flujo de información para apoyar la toma de decisiones estratégicas, generando una hoja de ruta de desarrollo de herramientas software que faciliten la gestión de información para el grupo de geomecánica del ICP.

\section{MATERIALES Y METODO}

“... la arquitectura empresarial se puede definir de dos posibles formas dependiendo del contexto en que se utilice, la primera a través de una descripción formal de un sistema o un plan detallado de un sistema en cuanto a sus componentes para guiar su implementación; o la segunda es con una estructura de componentes, sus interrelaciones, y los principios y guías que gobiernan su diseño y evolución en el tiempo" [5].

TOGAF es un Framework de Arquitectura Empresarial que proporciona los métodos y herramientas para ayudar en la aceptación, la producción, uso y mantenimiento de una arquitectura empresarial. Se basa en un modelo de proceso iterativo con el apoyo de mejores prácticas y un conjunto reutilizable de los activos de la arquitectura existente [5].

La metodología ADM es el núcleo del marco TOGAF. Es un enfoque iterativo de varias fases para desarrollar y utilizar una arquitectura empresarial para dar forma y gobernar los proyectos de implementación y transformación empresarial.

TOGAF define los principios como reglas y pautas generales para el uso e implementación de todos los recursos y activos de TI en la organización que forman la base para la toma de decisiones futuras de TI [5]. 
El análisis de brechas es un mecanismo para seleccionar el camino más apropiado para pasar de un estado actual a un estado objetivo. La transición entre la arquitectura de línea base y la arquitectura objetivo en un ejercicio de arquitectura consiste en establecer los elementos nuevos, los elementos que han sido modificados y/o eliminados y los elementos que permanecen sin alteraciones.

TOGAF define la Hoja de ruta de la arquitectura como una lista de componentes y módulos en una línea de tiempo. Cada componente o módulo identifica un grupo lógico de cambios o requerimientos necesarios para realizar la Arquitectura de destino [5].

La Figura 1 presenta un diagrama que detalla la ejecución del ejercicio de arquitectura empresarial donde se plantea la arquitectura actual y la arquitectura objetivo. Se realiza un análisis de brechas en cada dominio de la arquitectura, y con dicho análisis se construye una hoja de ruta, soportado en los objetivos de negocio, que permita generar proyectos que lleven la organización de la arquitectura actual a la arquitectura objetivo.

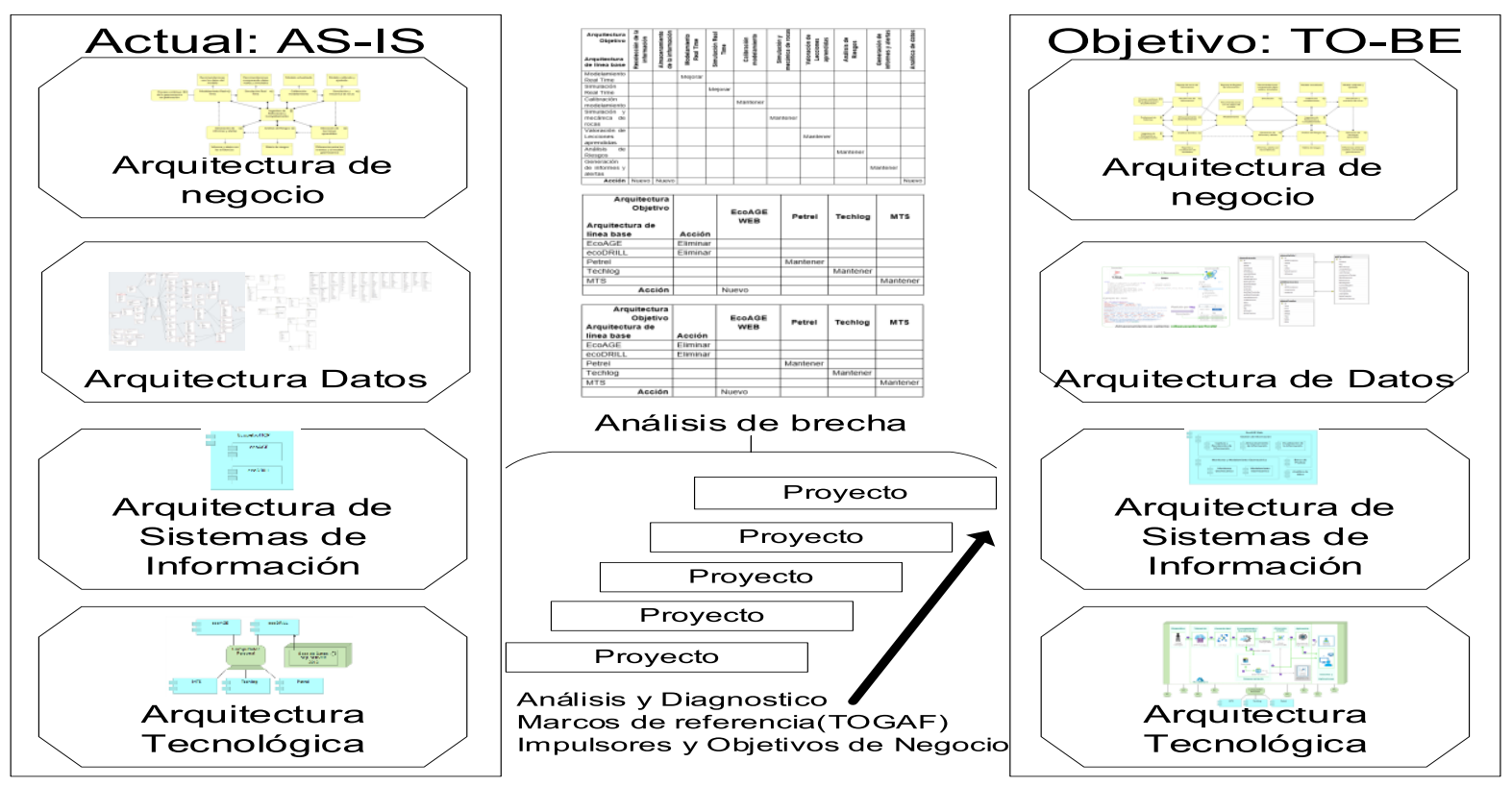

Figura 1. Ejercicio de arquitectura empresarial

Antes de iniciar el ejercicio de arquitectura empresarial se define el Marco de referencia de arquitectura empresarial adaptado (MRAE adaptado), el cual es un documento donde se presentan las fases del ADM de TOGAF que se aplicaron en el desarrollo del proyecto, detallando los entregables y artefactos a trabajar en cada fase.

En el diseño de la arquitectura empresarial se usaron las seis primeras fases de la metodología ADM (Preliminar, A, B, C, D y E) como se muestra en la Figura 2.

Las fases relacionadas en la arquitectura empresarial se ejecutan cíclicamente a través de cada uno de los tres dominios de arquitectura (arquitectura de negocio, arquitectura de sistemas de información y arquitectura tecnológica) y permiten asegurar que los requerimientos se aborden adecuadamente. 


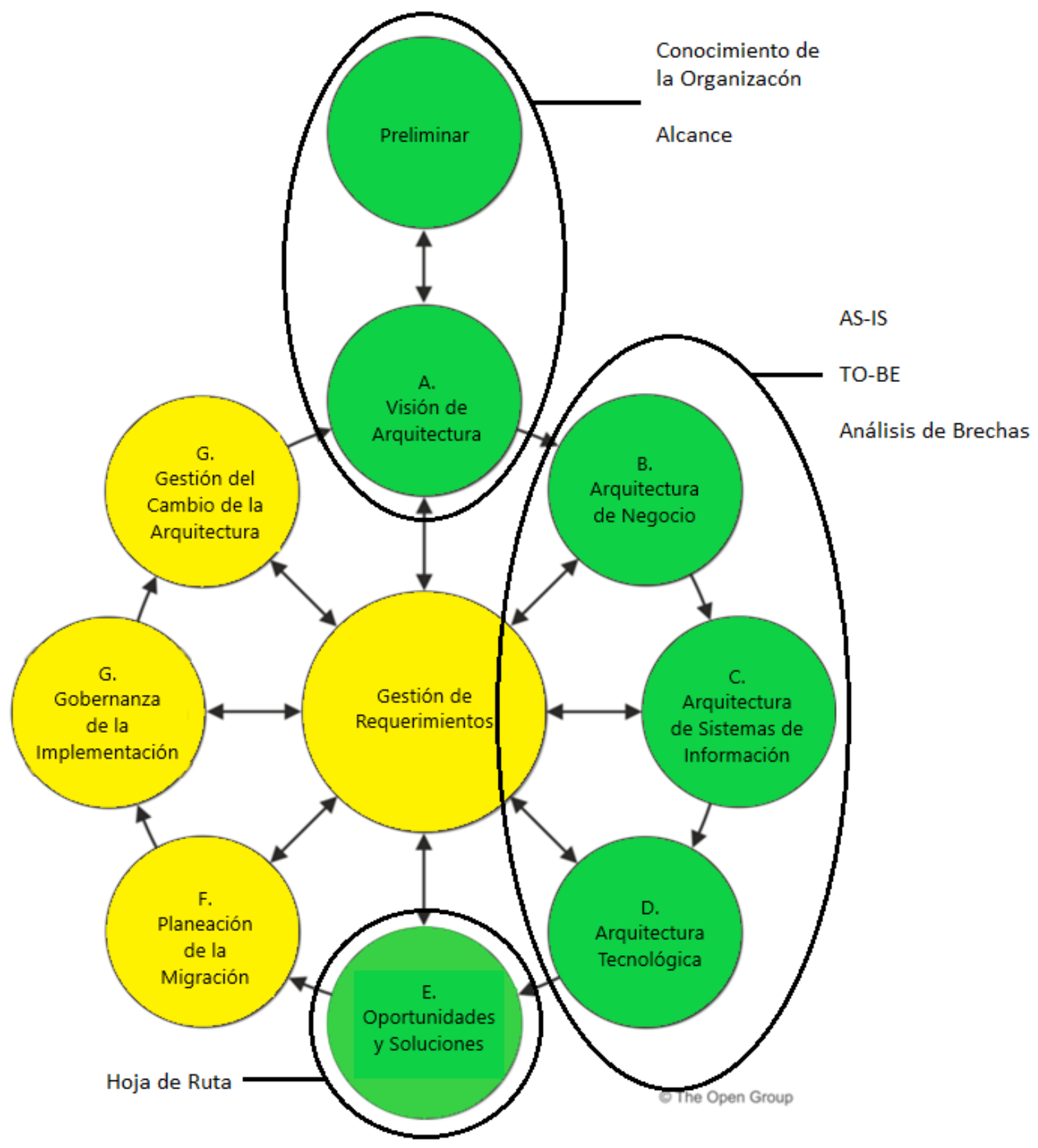

Figura 2. Fases del MRAE adaptado de [6]

\section{RESULTADOS}

A continuación, se detallan los resultados obtenidos en cada fase de la metodología ADM de arquitectura empresarial y contempladas en el MRAE adaptado.

\subsection{Fase Preliminar}

Esta fase se definen los principios generales y se identifica el modelo de la organización.

Para el presente proyecto se tomaron en cuenta los 21 principios propuestos por TOGAF dada la pertinencia de cada uno de estos, como se presenta en la Figura 3. 


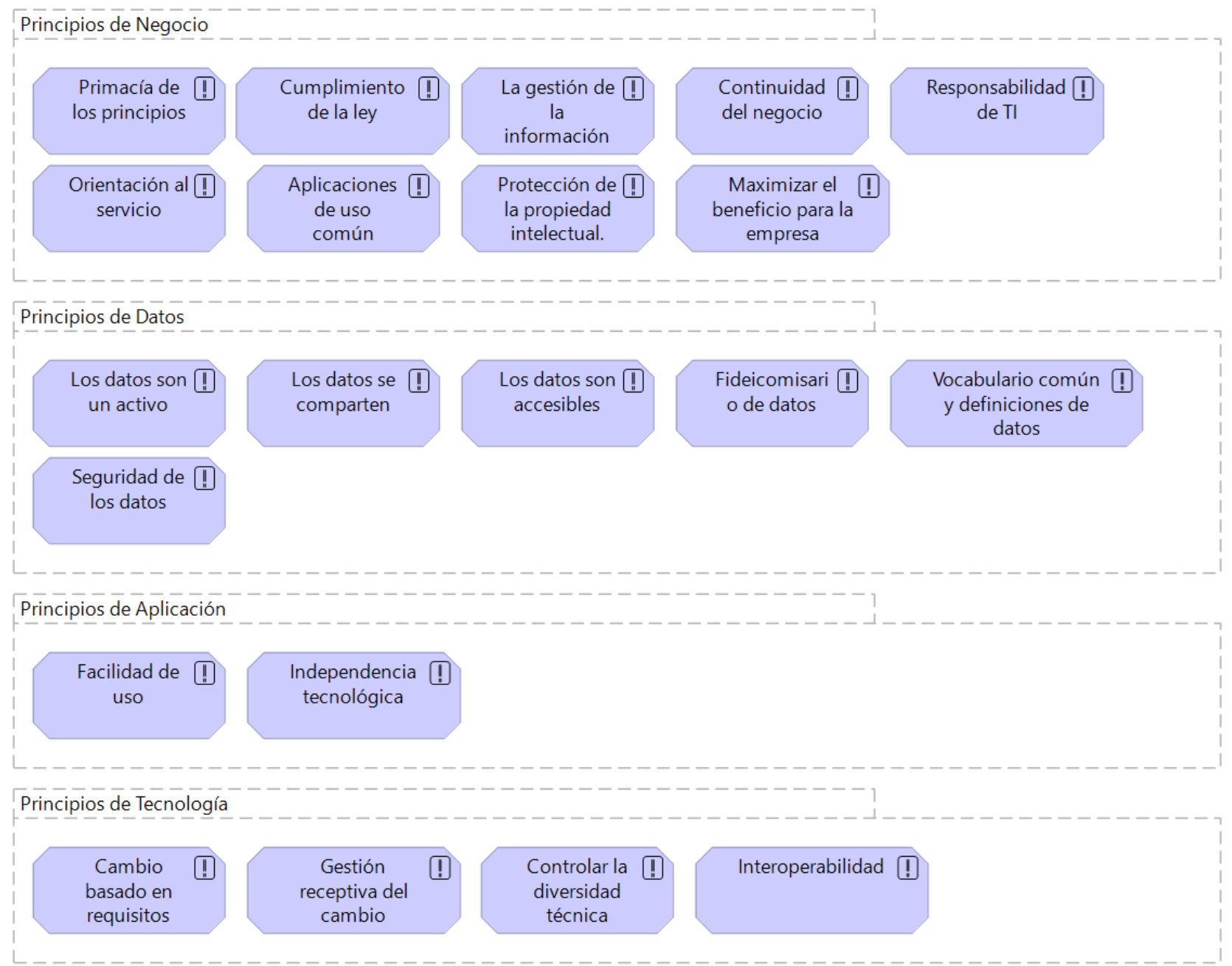

Figura 3. Principios de Arquitectura Empresarial

Los entregables de esta fase fueron:

- El modelo de organización de la Arquitectura Empresarial donde se identifican los roles y las responsabilidades dentro de la organización.

- La Solicitud de Trabajo de Arquitectura, documento con el cual se da inicio al desarrollo de la arquitectura y contiene información relacionando el resumen de la solicitud, los patrocinadores, misión y visión de la organización e Impulsores y objetivos de negocio

\subsection{Fase A: Visión de arquitectura}

En esta fase se preparan las siguientes fases a través de una representación general de las arquitecturas de línea base y objetivo.

El entregable de esta fase es la Declaración de trabajo de arquitectura en donde se establece el alcance y el enfoque que se utilizó para desarrollar la arquitectura empresarial.

En el diagrama motivacional, presentado en la Figura 4, se establecen los conceptos motivacionales de la arquitectura empresarial que guían y restringen el diseño de la misma y están representadas por objetivos, principios, requisitos y restricciones que representan el resultado deseado. 


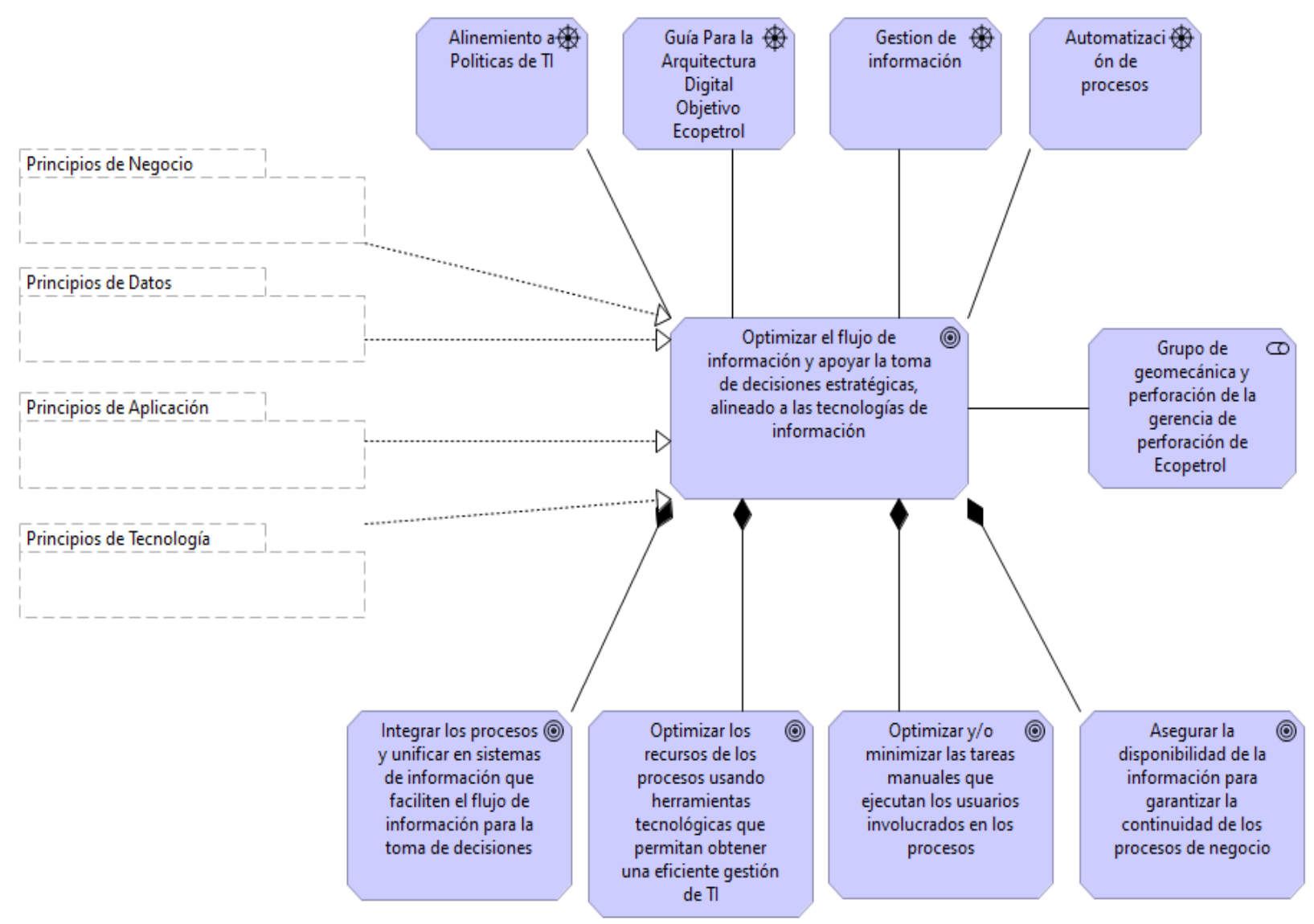

Figura 4. Diagrama motivacional del ejercicio de arquitectura empresarial

Un punto importante para una transformación exitosa es tener en cuenta la preparación que presenta el grupo de perforación para aceptar los cambios, identificar los riesgos y definir las acciones para limitarlos a través de planes de implementación y migración.

Para delimitar el alcance del ejercicio de arquitectura se establecieron tres dimensiones (amplitud, profundidad y dominios)

En la amplitud, se cubrió la necesidad de optimizar el flujo de información de los procesos relacionados a datos de perforación y completamiento del grupo de perforación del Instituto Colombiano del Petróleo.

En la profundidad, se ejecutó hasta la elaboración de una lista de proyectos con el posible orden de ejecución a fin de optimizar el flujo de información y apoyar la toma de decisiones estratégicas.

En los dominios, se abarcaron los 3 dominios de la arquitectura (arquitectura del negocio, arquitectura de sistemas de información y arquitectura de tecnología)

\subsection{Fase B: Arquitectura de negocio}

En esta fase del ciclo de arquitectura empresarial se identifican los principales procesos de negocio que sirven de base para el desarrollo de una arquitectura empresarial.

Las propuestas de mejora para la optimización del proceso actual de proceso continuo de la geomecánica en perforación incluyen una etapa de recolección de información teniendo en cuenta los diferentes protocolos gestionados y su posterior almacenamiento en bases de datos centralizadas para trabajo en tiempo real o manejo de históricos. 
A su vez, al estar centralizada la información se realizarán trabajos de analítica de datos a medida que se incremente la cantidad de información registrada.

En la Figura 5 se presentan los procesos de negocio en la arquitectura de línea base.

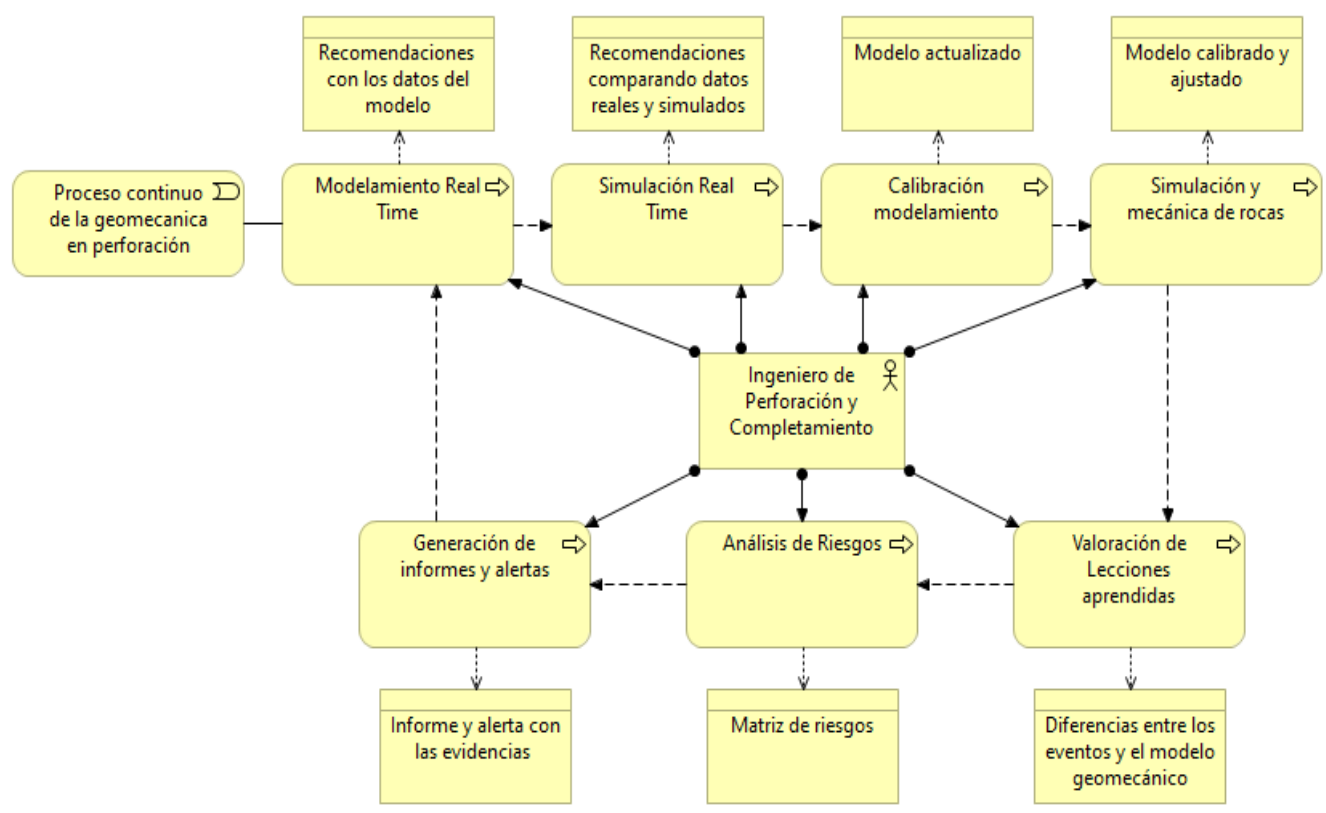

Figura 5. AS-IS de Negocio

En la Figura 6 se presentan los procesos de negocio en la arquitectura objetivo.

Al modificar la estructura del proceso continuo de la geomecánica en perforación implica la asignación de nuevos roles y la modificación de tareas para los roles existentes.

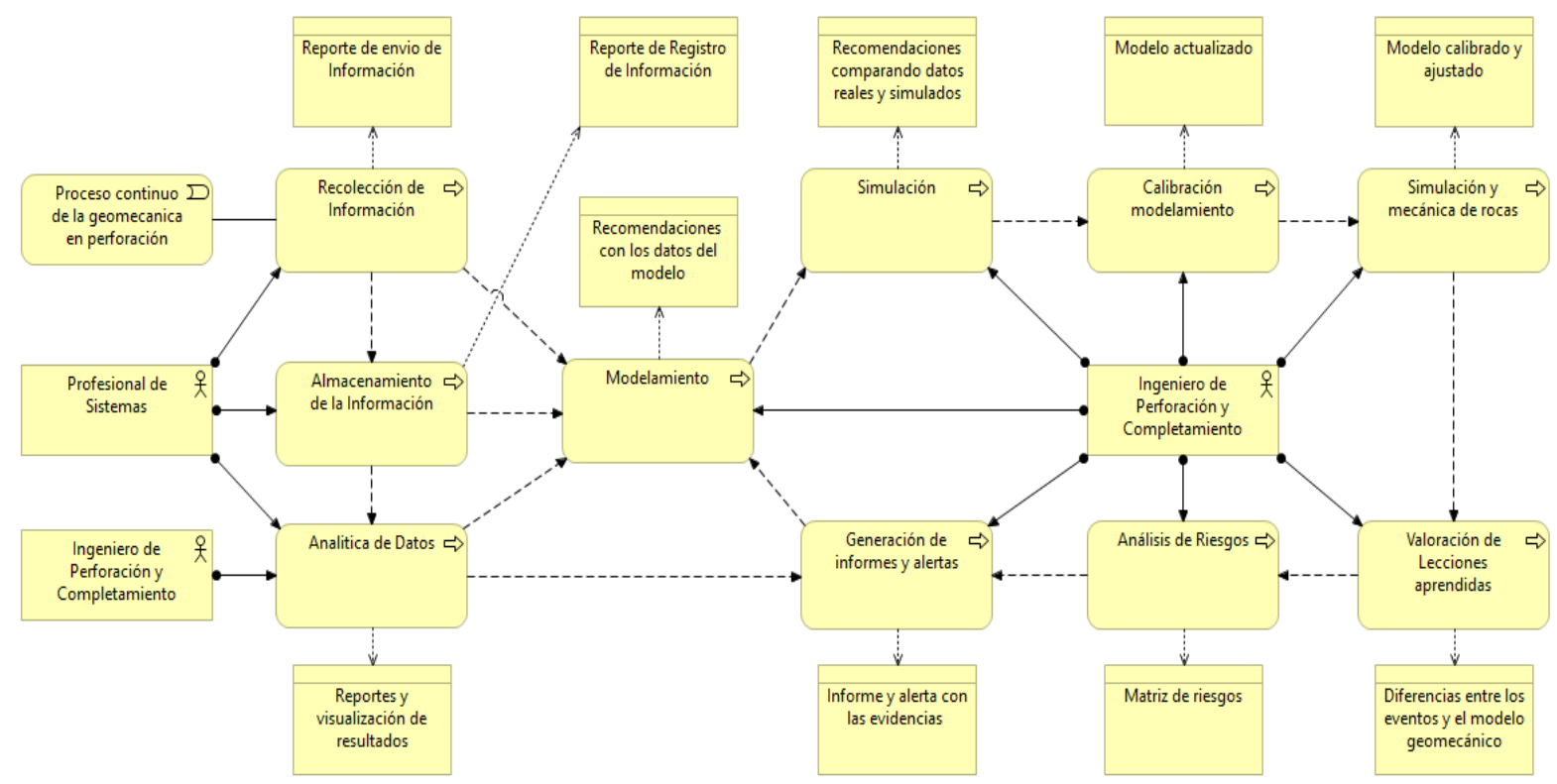

Figura 6. TO-BE de Negocio

En la fase de arquitectura de negocio se identificaron 5 brechas. 


\subsection{Fase C: Arquitectura de sistemas de información}

En esta fase se indica la forma en que los sistemas de información guiaran la obtención de los objetivos planteados en la visión de arquitectura.

En la Figura 7 se presentan los sistemas de información en la arquitectura de línea base.
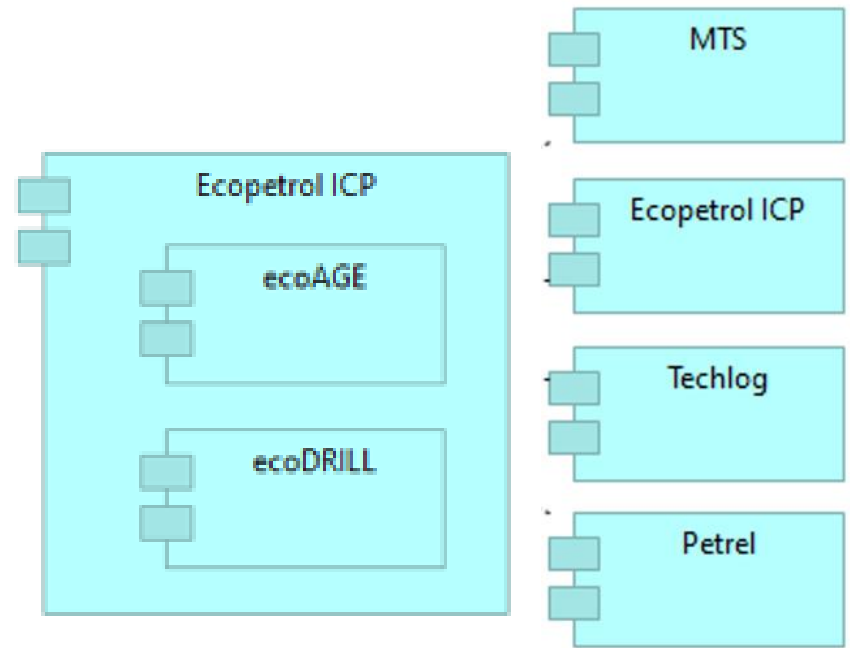

Figura 7. AS-IS de sistemas de información

En la Figura 8 se presentan los sistemas de información en la arquitectura objetivo.

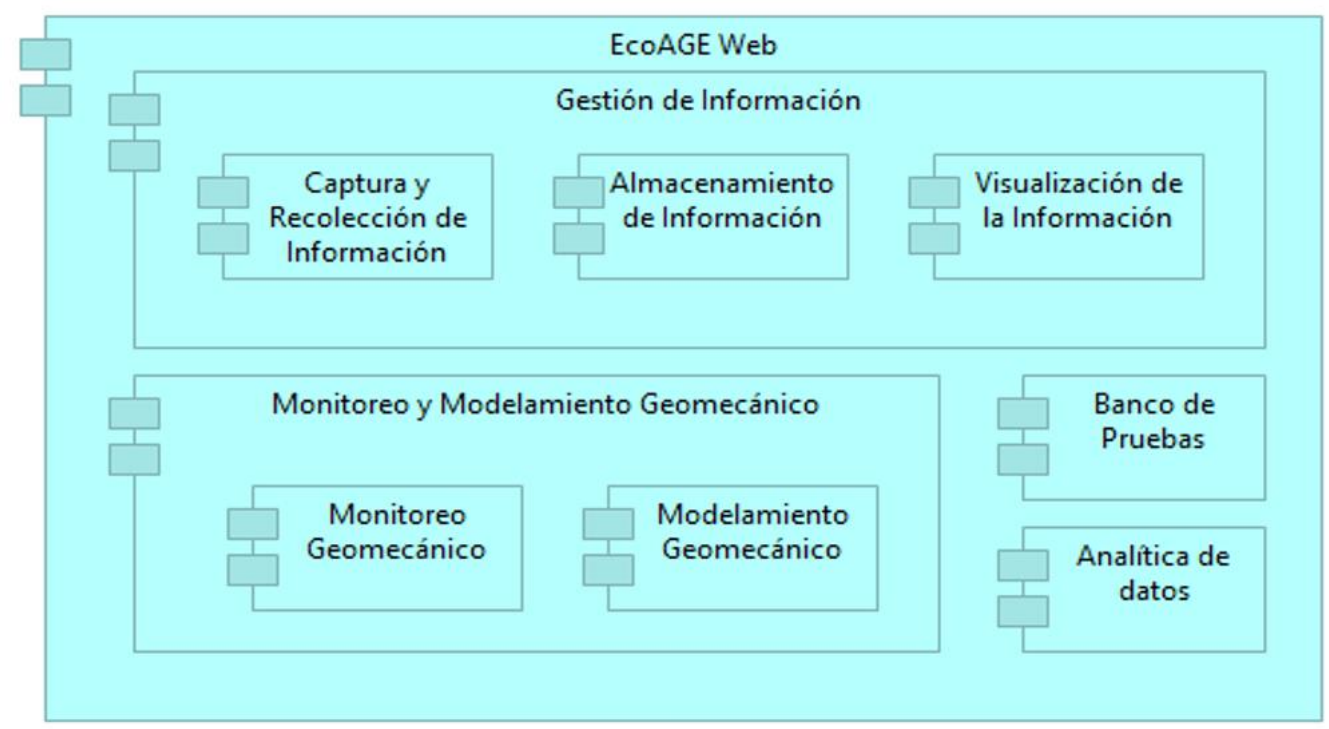

Figura 8. TO-BE de sistemas de información

En la fase de arquitectura de sistemas de información se identificaron 13 brechas.

\subsection{Fase D: Arquitectura tecnológica}

En esta fase se busca asociar componentes de la arquitectura de aplicaciones con los componentes de tecnología representados en software y hardware. 
En la Figura 9 se presentan los elementos de la arquitectura tecnológica en la arquitectura de línea base.

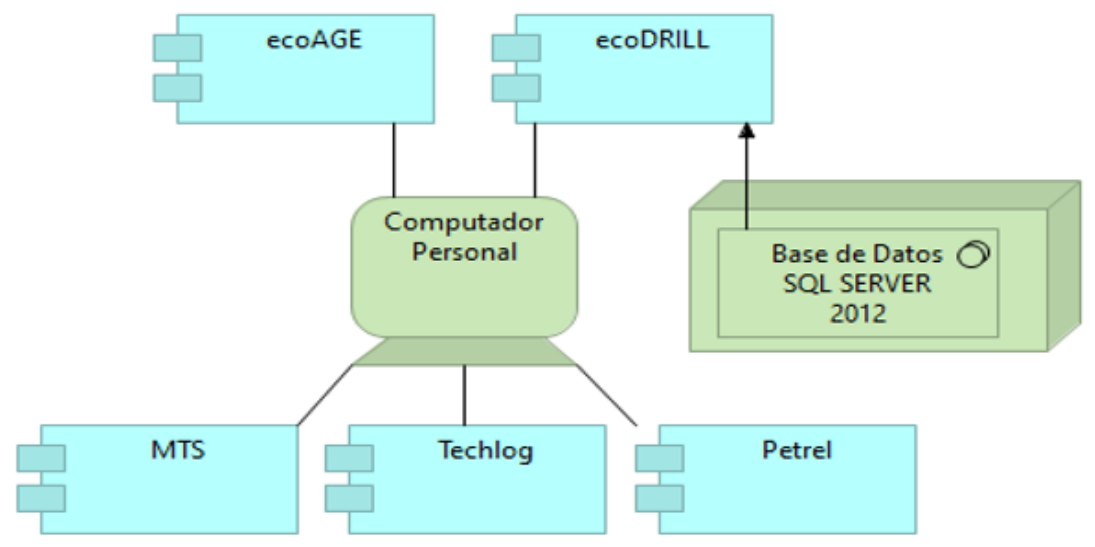

Figura 9. AS-IS de arquitectura tecnológica

En la Figura 10 se presentan los elementos de la arquitectura tecnológica en la arquitectura objetivo.

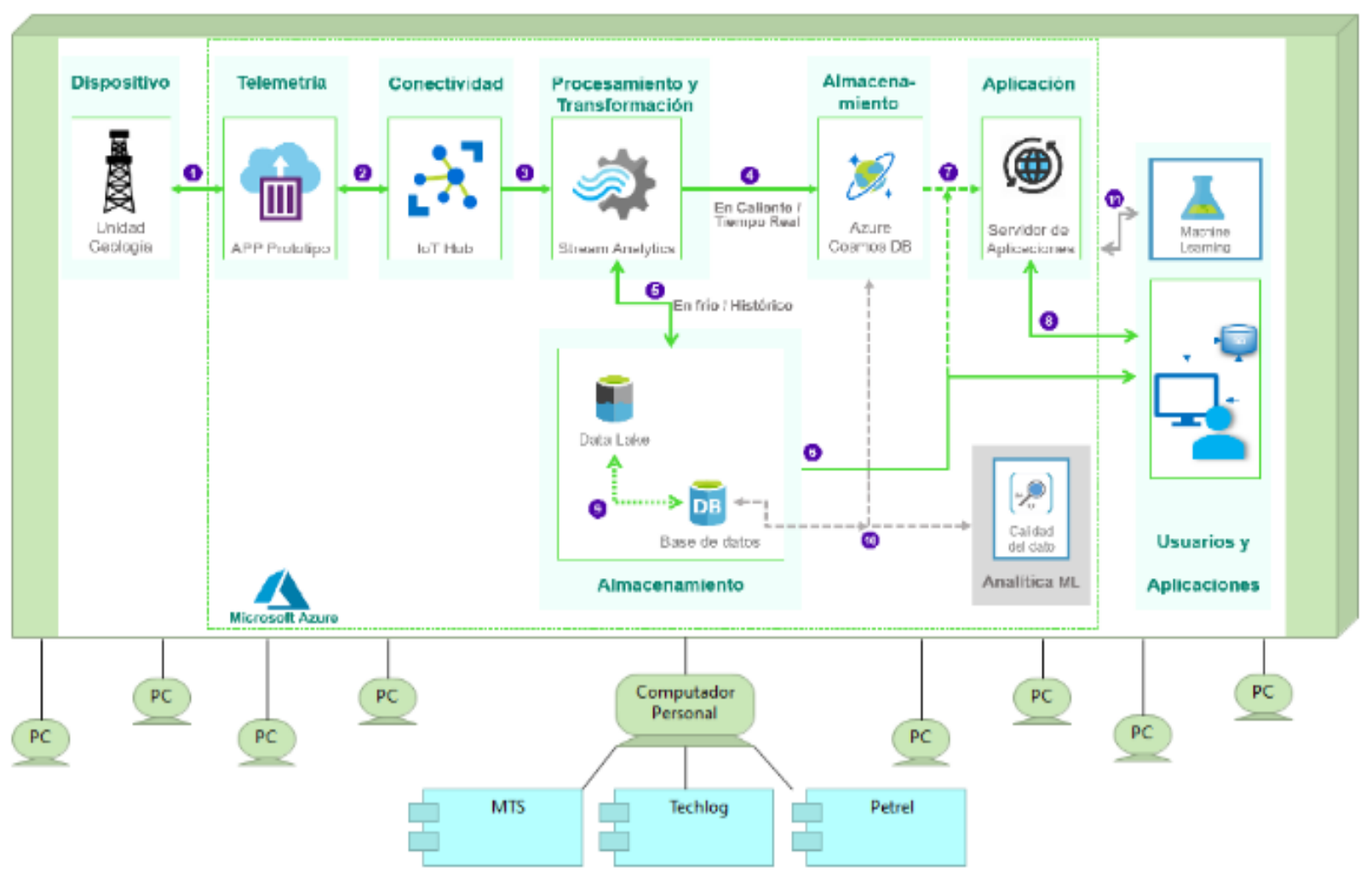

Figura 10. TO-BE de arquitectura tecnológica

En la fase de arquitectura tecnológica se identificaron 2 brechas.

\subsection{Fase E: Oportunidades y soluciones}

Teniendo en cuenta las brechas identificadas en cada una de las fases anteriores (fase de arquitectura de negocio, fase de arquitectura de sistemas de información y fase de arquitectura de tecnología), estas fueron agrupadas en iniciativas o proyectos que están orientados para conseguir los objetivos definidos en la visión de arquitectura. 
Con el análisis de brechas y el acompañamiento del grupo de perforación de Ecopetrol ICP se generaron 17 propuestas de mejora. En la Figura 11 se presenta la relación de proyectos y las brechas identificadas.

Las propuestas de mejora se integran en una suite especializada llamada EcoAGE.

\begin{tabular}{|c|c|c|c|c|c|c|c|c|c|c|c|c|c|c|c|c|c|}
\hline $\begin{array}{l}\text { ID Proyecto } \\
\text { ID GAP }\end{array}$ & $\bar{\alpha}$ & 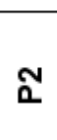 & ח2 & $\mathbb{a}$ & 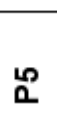 & $\mathscr{Q}$ & $\hat{n}$ & $\stackrel{\infty}{0}$ & ஜ & 움 & $\bar{\Sigma}$ & $\frac{\mathfrak{N}}{\Sigma}$ & $\frac{m}{\alpha}$ & $\frac{ \pm}{\Sigma}$ & $\frac{10}{\alpha}$ & $\frac{\varphi}{\alpha}$ & $\bar{\Sigma}$ \\
\hline GAP N1 & & & & & & & & & & & & & & & & & \\
\hline GAP N2 & & & & & & & & & & & & & & & & & \\
\hline GAP N3 & & & & & & & & & & & & & & & & & \\
\hline GAP N4 & & & & & & & & & & & & & & & & & \\
\hline GAP N5 & & & & & & & & & & & & & & & & & \\
\hline GAP S1 & & & & & & & & & & & & & & & & & \\
\hline GAP S2 & & & & & & & & & & & & & & & & & \\
\hline GAP S3 & & & & & & & & & & & & & & & & & \\
\hline GAP S4 & & & & & & & & & & & & & & & & & \\
\hline GAP S5 & & & & & & & & & & & & & & & & & \\
\hline GAP S6 & & & & & & & & & & & & & & & & & \\
\hline GAP S7 & & & & & & & & & & & & & & & & & \\
\hline GAP S8 & & & & & & & & & & & & & & & & & \\
\hline GAP S9 & & & & & & & & & & & & & & & & & \\
\hline GAP S10 & & & & & & & & & & & & & & & & & \\
\hline GAP S11 & & & & & & & & & & & & & & & & & \\
\hline GAP S12 & & & & & & & & & & & & & & & & & \\
\hline GAP S13 & & & & & & & & & & & & & & & & & \\
\hline GAP T1 & & & & & & & & & & & & & & & & & \\
\hline GAP T2 & & & & & & & & & & & & & & & & & \\
\hline
\end{tabular}

Figura 11. Relación de proyectos y brechas identificadas

La Figura 12 presenta la hoja de ruta para el año 2020 y los proyectos a realizarse.

\begin{tabular}{|c|c|c|c|c|c|c|c|c|c|c|c|c|c|c|}
\hline \multirow{2}{*}{ ID } & \multirow{2}{*}{\multicolumn{2}{|c|}{ Nombre }} & \multicolumn{12}{|c|}{2020} \\
\hline & & & 1 & 2 & 3 & 4 & 5 & 6 & 7 & 8 & 9 & 10 & 11 & 12 \\
\hline 1 & \multirow{5}{*}{ 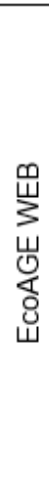 } & \begin{tabular}{|l|} 
Módulo captura de \\
información WITSML
\end{tabular} & & & & & & & & & & & & \\
\hline 2 & & $\begin{array}{l}\text { Módulo almacenamiento en } \\
\text { frio }\end{array}$ & & & & & & & & & & & & \\
\hline 3 & & $\begin{array}{l}\text { Módulo almacenamiento en } \\
\text { caliente }\end{array}$ & & & & & & & & & & & & \\
\hline 4 & & $\begin{array}{l}\text { Módulo visualización en } \\
\text { tiempo real }\end{array}$ & & & & & & & & & & & & \\
\hline 5 & & $\begin{array}{l}\text { Módulo visualización de } \\
\text { históricos }\end{array}$ & & & & & & & & & & & & \\
\hline
\end{tabular}

Figura 12. Hoja de ruta para el año 2020

La Figura 13 presenta la hoja de ruta para los años 2021-2025 y los proyectos a realizarse. 


\begin{tabular}{|c|c|c|c|c|c|c|c|}
\hline ID & & Nombre & 2021 & 2022 & 2023 & 2024 & 2025 \\
\hline 6 & \multirow{11}{*}{ 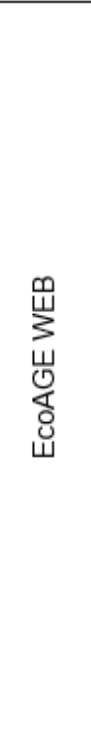 } & Módulo monitoreo geo mecánico & & & & & \\
\hline 7 & & Módulo visualización de curvas & & & & & \\
\hline 8 & & Módulo datos experimentales & & & & & \\
\hline 9 & & Módulo loT Edge & & & & & \\
\hline 10 & & Módulo banco de pruebas & & & & & \\
\hline 11 & & $\begin{array}{l}\text { Módulo gemelo digital banco de } \\
\text { pruebas }\end{array}$ & & & & & \\
\hline 12 & & $\begin{array}{l}\text { Módulo captura de información } \\
\text { WITSML no estándar }\end{array}$ & & & & & \\
\hline 13 & & Módulo captura de información WITSO & & & & & \\
\hline 14 & & $\begin{array}{l}\text { Módulo flujos de trabajo de } \\
\text { modelamiento geo mecánico }\end{array}$ & & & & & \\
\hline 15 & & $\begin{array}{l}\text { Módulo flujos de trabajo de monitoreo } \\
\text { geo mecánico en tiempo real }\end{array}$ & & & & & \\
\hline 16 & & $\begin{array}{l}\text { Módulo flujos de trabajo de análisis de } \\
\text { datos experimentales }\end{array}$ & & & & & \\
\hline 17 & 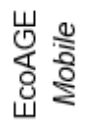 & Módulo operaciones de perforación & & & & & \\
\hline
\end{tabular}

Figura 13. Hoja de ruta para los años 2021-2025

\section{CONCLUSIONES}

Resultó valioso ejecutar el ejercicio de arquitectura empresarial porque además de generar una solución inmediata a una necesidad específica, permitió identificar todos los aspectos de la organización que intervienen en la problemática.

Los principios de arquitectura son elementos que guían la evolución de la organización, no pueden verse como un documento. Conocer los principios ha permitido que las personas en el grupo entiendan y consideren criterios a tener en cuenta al plantear los proyectos y procesos, centrados en la misión y visión de la organización.

El análisis de brechas entre el estado actual y el estado objetivo en cada dominio de la arquitectura permitió tener criterios para definir claramente los proyectos propuestos en la hoja de ruta.

Se logro concientizar a la directiva sobre la importancia de contar con una Arquitectura Empresarial que permita el alineamiento de los procesos de negocio con los objetivos de la organización. Actualmente, el grupo de perforación. se encuentra interesado en la implementación de la presente propuesta de solución que le permitirá mejorar sus procesos de negocio soportados en tecnología de información.

Se recomienda la validación y posterior implementación de la arquitectura propuesta, junto con el uso de buenas prácticas con el objeto de obtener mejoras significativas en los servicios y procesos, todo esto para disipar las brechas analizadas.

Se recomienda elaborar un plan detallado de implementación y migración sobre la hoja de ruta para el periodo 2021-2025 que presente a profundidad la ejecución de los proyectos que permita partir desde la arquitectura de la línea base hacia la arquitectura destino para poder realizar una adecuada estimación de recursos, costos, y evaluación de riesgos. 


\section{AGRADECIMIENTOS}

Los autores expresan sus agradecimientos a la Universidad Autónoma de Bucaramanga y al Instituto Colombiano del Petróleo por apoyar el desarrollo de este proyecto y por poner a disposición los recursos necesarios para la realización del ejercicio de arquitectura empresarial.

\section{REFERENCIAS BIBLIOGRÁFICAS}

[1] Centro para Soluciones de Energía de Deloitte. De los bytes a los barriles. La transformación digital en el segmento UpStream del sector de los hidrocarburos. Cuadernos de Energía № 54 , páginas 5569 , febrero de 2018.

[2] Kashif, M. y Khudiri, M. Customized real-time data solution improves efficiency, decision making. Abril de 2017.

[3] Instructivo Para Arquitectura y Gobierno en Microsoft Azure. GDI-I-006. Bogotá, Gerencia de Información. Vicepresidencia Digital Ecopetrol, 2019.

[4] Guía para la arquitectura digital objetivo Ecopetrol. GDI-G-028. Bogotá, Vicepresidencia Digital Ecopetrol, 2019.

[5] The TOGAF Standard, Versión 9.2. Disponible en: https://pubs.opengroup.org/architecture/togaf92doc/arch/ [consultado el 15 de diciembre de 2019].

[6] Gelvis, J. Propuesta de arquitectura empresarial en la gestión de TI aplicado en el grupo de geomecánica y perforación de la gerencia de perforación de Ecopetrol con base en marcos de referencia de arquitectura empresarial. [Tesis de Maestría]. Bucaramanga, Universidad Autónoma de Bucaramanga, 2020. 